\title{
Sourcing local produce
}

The population of cortical glial cells expands greatly during the first 3 weeks after birth in rodents. Progenitor cells in the subventricular zone (SVZ) were thought to be the source of the new cells postnatally, but Ge et al. now show that the expansion is, in fact, due to local proliferation of astrocytes in the cortex.

The authors first showed that in mice, SVZ progenitor cells labelled with green fluorescent protein (GFP) on postnatal day (PND) 0-2 generated GFP-expressing $\left(\mathrm{GFP}^{+}\right)$cells that mainly remained in the SVZ or migrated to deep cortical layers.

dividing astrocytes only gave rise to astrocytes and not to other types of cells This suggested that glial cells in the upper cortical layers must have a different - probably local — source. To investigate this possibility, the authors injected, on PND 0-6, a retrovirus in cortical layers I-IV that induced GFP expression specifically in proliferating cells in these layers.

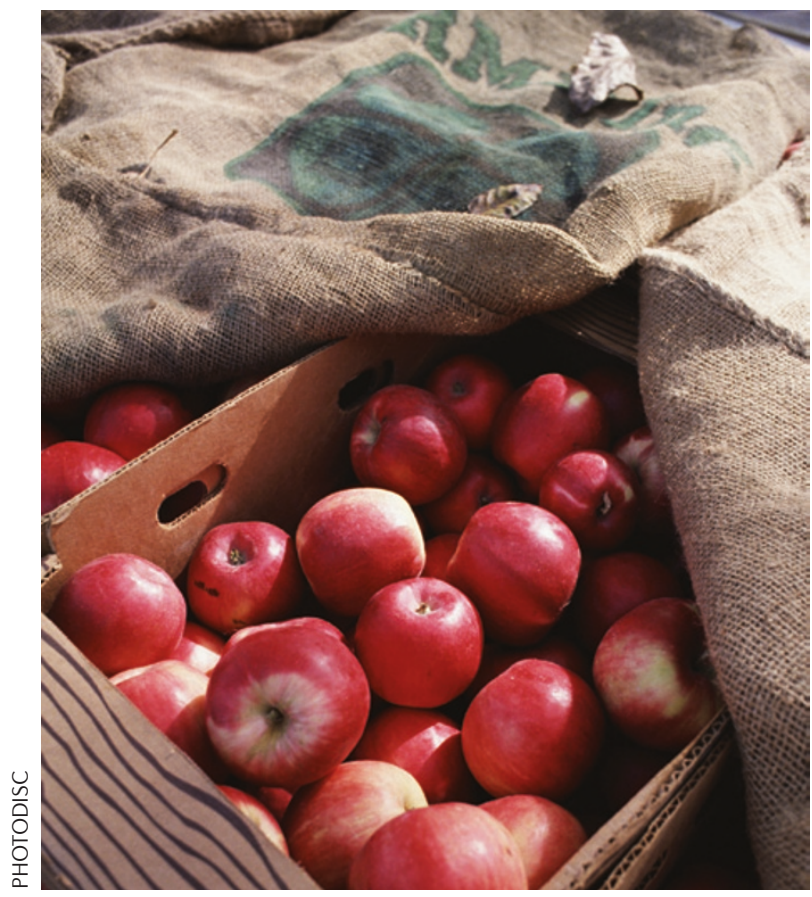

They then identified the progeny of these cells 1 week later. Most of the $\mathrm{GFP}^{+}$cells were astrocytes; moreover, about half of all astrocytes in these layers were $\mathrm{GFP}^{+}$, indicating that a large proportion of astrocytes in the postnatal brain are locally generated.

To establish which cells give rise to the new astrocytes, the authors labelled actively dividing cells in acute brain slices with Hoechst dye and identified the labelled cells by their electrophysiological responses in patch-clamp recordings. One population consisted of NG2 cells, which are known to produce few astrocytes in the postnatal brain. The other population consisted of astrocytelike cells. These cells had complex cell morphology, astrocyte-like ion-channel properties and formed gap junctions with non-dividing astrocytes. This finding suggests that the dividing astrocyte-like cells are differentiated astrocytes rather than undifferentiated progenitor cells.

The authors further showed that dividing astrocytes only gave rise to astrocytes and not to other types of cells. In addition, their daughter cells were functionally integrated in existing cellular networks: they formed gap junctions with other astrocytes, formed endfeet on blood vessels and could clear glutamate from synapses.

These findings provide convincing evidence that the postnatal expansion of glia is largely due to local production of astrocytes, and may have implications for our understanding of the pathophysiology of some forms of glioma, a brain tumour that originates from over-proliferation of glial cells.

Leonie Welberg

ORIGINAL RESEARCH PAPER Ge, W.-P. et al.

Local generation of glia is a major astrocyte source in postnatal cortex. Nature 28 Mar 2012 (doi:10.1038/nature10959) 